\title{
Increased protein expression levels of pCREB, BDNF and SDF-1/CXCR4 in the hippocampus may be associated with enhanced neurogenesis induced by environmental enrichment
}

\author{
XIAO QIAN ZHANG ${ }^{1}$, JING WEI MU ${ }^{2}$, HUI BIN WANG ${ }^{1}$, JUKKA JOLKKONEN ${ }^{3}$, TING TING LIU ${ }^{1}$, \\ TING XIAO ${ }^{4,5}$, MEI ZHAO ${ }^{6}$, CHAO DONG ZHANG $^{1}$ and CHUAN SHENG ZHAO ${ }^{1}$ \\ ${ }^{1}$ Department of Neurology, The First Affiliated Hospital of China Medical University, Shenyang, Liaoning 110001; \\ ${ }^{2}$ Department of Neurology, The People's Hospital of Liaoning, Shenyang, Liaoning 110016, P.R. China; \\ ${ }^{3}$ Institute of Clinical Medicine-Neurology, University of Eastern Finland, 70211 Kuopio, Finland; \\ ${ }^{4}$ Department of Dermatology, The First Affiliated Hospital, China Medical University; \\ ${ }^{5}$ Key Laboratory of Immunodermatology, Ministry of Health, Ministry of Education, Shenyang, Liaoning 110001; \\ ${ }^{6}$ Department of Cardiology, The Shengjing Affiliated Hospital, China Medical University, \\ Shenyang, Liaoning 110004, P.R. China
}

Received August 29, 2015; Accepted May 31, 2016

DOI: $10.3892 / \mathrm{mmr} .2016 .5470$

\begin{abstract}
Brain plasticity is very sensitive to the environment. Certain neurotrophic factors and neurotransmitter receptors, including brain-derived neurotrophic factor (BDNF), cyclic adenosine monophosphate response element-binding protein (CREB), stromal cell-derived factor-1 (SDF-1) and its specific receptor, $\mathrm{C}-\mathrm{X}-\mathrm{C}$ motif chemokine receptor 4 (CXCR4), are important in neurogenesis in adult animals. In the present study, the effects of environmental enrichment (EE) on neurogenesis in the dentate gyrus (DG) and subventricular zone (SVZ), and the protein expression levels of BDNF, CREB, SDF-1 and CXCR4 were investigated. Adult rats were randomly assigned as controls or underwent EE for 30 days. Subsequently, immunofluorescence staining was used to analyze cell proliferation in the DG and SVZ, and the differentiation and survival of newly-formed cells in the hippocampus. The protein expression levels of BDNF, phosphorylated CREB (pCREB), protein kinase A catalytic subunit $\alpha$, SDF-1 and CXCR4 in the hippocampus were assayed by western blotting. Cognitive function was assessed in a Morris water maze. EE improved cognitive function, and increased the proliferation, differentiation and survival of newly-formed neurons in the DG of
\end{abstract}

Correspondence to: Professor Chao Dong Zhang or Professor Chuan Sheng Zhao, Department of Neurology, The First Affiliated Hospital of China Medical University, 155 North Nanjing Street, Heping, Shenyang, Liaoning 110001, P.R. China

E-mail: chaodongzhang@126.com

E-mail: cszhao@mail.cmu.edu.cn

Key words: environmental enrichment, neurogenesis, cognitive function, stromal cell-derived factor-1 adult rats; however, EE did not activate neurogenesis in the SVZ. Furthermore, EE enhanced the protein expression levels of BDNF, pCREB, SDF-1 and CXCR4 in the hippocampus. These results provide a theoretical basis to explain the beneficial effects of EE on healthy, adult rats.

\section{Introduction}

Ongoing neurogenesis in adults is now an accepted and well-characterized phenomenon in the mammalian brain. In the subgranular zone (SGZ) of the dentate gyrus (DG) of the hippocampus and the subventricular zone (SVZ) of the olfactory bulb, the adult rodent brain regularly produces newly-formed cells that differentiate into neurons, astrocytes and oligodendrocytes (1). The process of adult neurogenesis is influenced by numerous physiological and pathological stimuli, including brain injury, neurotrophic factors or chemokines, and the environment (2-5).

Environmental enrichment (EE) is defined as a combination of 'complex inanimate objects and social stimulation' (6). Compared with standard housing conditions, EE provides enhanced stimulation of the cognitive, sensory and motor systems in the brains of laboratory animals (7-10). Current evidence demonstrates that EE increases hippocampal neurogenesis, improves cognition and promotes behavioral recovery following a brain injury in animals (11-13). However, the effects of EE on neurogenesis in the DG and SVZ remain to be fully resolved. Furthermore, the mechanisms underlying EE exposure-induced hippocampal neurogenesis remain to be elucidated.

Brain-derived neurotrophic factor (BDNF) is an important neuronal growth factor in the brain that promotes neuronal maturation and neurogenesis by activating cyclic adenosine monophosphate (cAMP) response element-binding protein (CREB) and protein kinase A (PKA) (14). Increasing evidence 
suggests that BDNF and phosphorylated CREB (pCREB) are key factors in the regulation of hippocampal neurogenesis and improved cognition, which are observed following EE (1,13,15-17). Stromal cell-derived factor-1 (SDF-1) is a CXC chemokine produced by bone marrow stromal cells. SDF-1 and its specific receptor, $\mathrm{C}-\mathrm{X}-\mathrm{C}$ motif chemokine receptor 4 (CXCR4), which stimulates the cAMP-mediated signaling pathway, are important in the regulation of the proliferation and differentiation of neural precursors (18-22).

In the present study, the impact of the provision of daily EE for 30 days on neurogenesis in the DG and SVZ, as well as on cognitive function in healthy, adult rats was examined. In addition, the protein expression levels of BDNF, pCREB, PKA catalytic subunit $\alpha$ (PKA C- $\alpha$ ), SDF-1 and CXCR4 in the hippocampus of rats housed in the presence or absence of $\mathrm{EE}$ were determined.

\section{Materials and methods}

Animals and EE. Adult male Wistar rats (weight, 220-250 g) from the Experimental Animal Center of China Medical University (Shenyang, China) were randomly assigned to two groups: Standard environment $(\mathrm{SE} ; \mathrm{n}=24)$ and $\mathrm{EE}(\mathrm{n}=24)$. The EE animals were housed for 30 days in a large cage $(841 \times 565 \times 526 \mathrm{~mm})$ containing a variety of toys, including houses, mazes, wheels, chains, sinks, swings, ladders and balls; the toys were changed once or twice per week. The SE group was housed in standard vivarium cages $(30 \times 20 \times 15 \mathrm{~mm})$ without toys. There were 3-4 rats in each standard cage and 8-12 rats in the EE cage. All rats were housed under a temperature-regulated environment with a 12-h light/dark cycle and free access to food and water. All procedures were approved by the Institutional Animal Care and Use Committee of China Medical University [Shenyang, China; ref. SCXK (Liao) 2008-0005].

Morris water maze test. Spatial learning was analyzed with a match-to-place version of the Morris water maze test on days 31-33, as described previously (23). Following the assessment (day 33), a probe trial of $60 \mathrm{sec}$ in the absence of the platform was performed to estimate the ability of the rats to recall the location of the platform by measuring the number of passings over its previous location. Swimming routes were monitored with the SLY-WMS video tracking system (Beijing Sunny Instruments Co., Ltd., Beijing, China).

5-Bromo-2-deoxyuridine ( $\mathrm{Brd} U$ ) labeling. To examine the rate of cell differentiation and survival, one cohort of rats ( $\mathrm{n}=12$ per group) was injected i.p. with $\mathrm{BrdU}$ (100 mg/kg; Sigma-Aldrich, St. Louis, MO, USA) once a day on days 11-15 and sacrificed on day 36 . In order to investigate the rate of cell proliferation, one cohort of rats ( $n=6$ per group) received injections of BrdU twice daily on days 34-35 prior to sacrifice on day 36.

Tissue preparation. The rats were transcardially perfused with normal saline and $4 \%$ paraformaldehyde under anesthesia, following which the brains were removed immediately and postfixed in $4 \%$ paraformaldehyde overnight at $4^{\circ} \mathrm{C}$. Brains were then placed in a $30 \%$ sucrose solution. When the tissues had sunk to the bottom of the container, they were removed, placed in optimal cutting temperature compound (Sakura Finetek USA, Inc., Torrance, CA, USA) and snap frozen. A series of contiguous $40-\mu \mathrm{m}$-thick coronal sections were cut on a cryotome (Leica Microsystems $\mathrm{GmbH}$, Wetzlar, Germany). A total of five coronal sections were selected from every sixth section between bregma level -2.8 and $-4.0 \mathrm{~mm}$ through the dorsal hippocampus or between bregma level +0.96 and $-0.24 \mathrm{~mm}$ through the SVZ in each rat prior to immunostaining.

Immunofluorescence staining. As described previously (24), DNA was denatured and the incorporated BrdU was detected immunologically using a sheep anti-BrdU antibody (1:500; catalog no. ab1893; Abcam, Cambridge, MA, USA). The following antibodies were used for phenotyping in combination with anti-BrdU: Guinea pig anti-doublecortin (DCX; 1:1,000; catalog no. AB5910; EMD Millipore, Billerica, MA, USA), mouse anti-neuronal nuclei (NeuN; 1:500; catalog no. MAB377; Chemicon; EMD Millipore) or rabbit anti-glial fibrillary acidic protein (GFAP; 1:1,000; catalog no. ab7260; Abcam). The sections were incubated in the appropriate secondary antibodies: Alexa Fluor 594 donkey anti-sheep IgG (catalog no. A11016), Alexa Fluor 488 goat anti-guinea pig IgG (catalog no. A11073) or Alexa Fluor 488 goat anti-rabbit IgG (catalog no. A11034), all diluted 1:500 and purchased from Thermo Fisher Scientific, Inc. (Waltham, MA, USA), prior to immunofluorescent assessment.

Quantification. Positively stained cells were counted by an experimenter blinded to the details of the study. The immunofluorescence images of BrdU-, BrdU/DCX-, BrdU/NeuN- and BrdU/GFAP-positive cells in the DG, and BrdU- and BrdU/DCX-positive cells in the SVZ were visualized on a fluorescent microscope with a 20x objective lens (Olympus Corporation, Tokyo, Japan). The numbers of positive cells in five sections per rat were counted with ImageJ software version 1.48 (National Institutes of Health, Bethesda, MD, USA) and the mean number was calculated. Only cells in which a BrdU-positive nucleus was co-expressed with DCX, NeuN or GFAP were deemed to be newly-formed neurons, mature neurons or astrocytes, respectively. For analysis of dendritic complexity, 18-20 DCX-positive cells from each section were randomly selected and visualized using a confocal system (Leica SP2; Leica Microsystems $\mathrm{GmbH}$ ) with a multi-track configuration. Dendritic length was assessed with FIJI (a distribution of ImageJ; www.fiji.sc/) using the Simple Neurite Tracer plugin. Total dendritic length was assessed for each neuron by tracing main dendritic processes extending from the soma and their branches.

Western blotting. Western blotting was performed on day 34 following SE or EE. Hippocampal proteins of eight rats per group were extracted using ice-cold radioimmunoprecipitation assay buffer (Beyotime Institute of Biotechnology, Haimen, China), and protein concentrations were measured using a standard Bradford assay (Beyotime Institute of Biotechnology). Protein samples $(40 \mu \mathrm{g})$ were separated on $10 \%$ SDS-PAGE gels at $80 \mathrm{~V}$ for $5 \mathrm{~h}$ and transferred onto polyvinylidene difluoride (PVDF) membranes (Beyotime Institute 
of Biotechnology). The PVDF membranes were blocked with $5 \%$ bovine serum albumin (Sigma-Aldrich) for $2 \mathrm{~h}$ at room temperature prior to antibody incubation. The following primary antibodies were used: Rabbit anti-PKA C- $\alpha$ (1:1,000; catalog no. 5842), rabbit anti-pCREB (1:1,000; catalog no. 9198) and rabbit anti-CREB (1:1,000; catalog no. 9197), all Cell Signaling Technology, Inc. (Danvers, MA, USA); rabbit anti-BDNF (1:500; catalog no. ab108319), rabbit anti-SDF-1 (1:1,000; catalog no. ab9797) and rabbit anti-CXCR4 (1:500; catalog no. ab197203), from Abcam; and mouse anti- $\beta$-actin (1:1,000; catalog no. sc-47778; Santa Cruz Biotechnology, Inc., Dallas, TX, USA). The secondary antibodies were horseradish peroxidase-conjugated goat anti-rabbit IgG $(1: 2,000$; catalog no. ZB2301; ZsBio, Beijing, China) and goat anti-mouse IgG (1:2,000; catalog no. ZB2305; ZsBio). Proteins were observed with an enhanced chemiluminescence reagent kit (Beyotime Institute of Biotechnology). The densities of the protein signals were quantified using Image-Pro Plus version 6.0 (Media Cybernetics, Inc., Rockville, MD, USA).

Statistical analysis. Statistical analyses were performed using SPSS software version 20 (IBM SPSS, Armonk, NY, USA). Data were analyzed using the independent-samples $t$-test or repeated-measures analysis of variance. Data are presented as the mean \pm standard error of the mean and $\mathrm{P}<0.05$ was considered to indicate a statistically significant difference.

\section{Results}

Quantifying the proliferation and dendritic length of newly-formed neurons in the DG. Double immunofluorescence staining of BrdU and DCX (an immature neuron maker) was conducted to examine the identity of the proliferating cells. In the DG, the numbers of BrdU-positive and BrdU/DCX double-positive cells (Fig. 1A and B) were significantly increased in EE compared with SE rats [25.34 1.05 vs. $14.51 \pm 1.06(\mathrm{P}<0.001)$ and $15.53 \pm 0.77$ vs. $7.50 \pm 0.60(\mathrm{P}<0.001)$, respectively]. The effect of EE on apical dendritic development was assessed by measuring the total dendritic length of the DCX-positive cells in the DG (Fig. 1C and D). In contrast to the SE group $(330.31 \pm 8.59 \mu \mathrm{m})$, DCX-positive cells in the EE group exhibited a significant increase in apical dendritic length $(981.95 \pm 51.90 \mu \mathrm{m} ; \mathrm{P}<0.001)$.

Quantifying the proliferation of newly-formed neurons in the $S V Z$. As presented in Fig. 1E and F, no statistically significant differences were observed between the EE and SE rats in the numbers of BrdU-positive and BrdU/DCX double-positive cells in the SVZ $[232.91 \pm 19.97$ vs. $201.04 \pm 21.75(\mathrm{P}=0.299)$ and $60.93 \pm 3.07$ vs. $58.29 \pm 2.43(\mathrm{P}=0.510)$, respectively].

Quantification of the differentiation and survival of the neuroblasts generated in the DG. The differentiation of newly-formed cells in the DG was assessed by double-staining of BrdU and NeuN (a mature neuron marker) or GFAP (an astrocyte marker). The long-term survival of newly-formed neurons was determined by analysis of BrdU associated with the neuronal marker, NeuN (Fig. 2A and B). The numbers of BrdU-positive and BrdU/NeuN double-positive cells were markedly increased in the EE group in comparison with the SE group $[18.11 \pm 1.07$ vs. $13.72 \pm 1.36(\mathrm{P}=0.025)$ and $11.54 \pm 0.80$ vs. $8.67 \pm 0.78(\mathrm{P}=0.016)$, respectively]. However, no significant differences were observed between the numbers of BrdU/GFAP double-positive cells (Fig. 2C and D) in the SE and $\mathrm{EE}$ groups $(4.14 \pm 0.52$ vs. $5.11 \pm 0.61 ; \mathrm{P}=0.206)$.

Protein expression levels of PKA C- $\alpha$, pCREB, BDNF, SDF-1 and $C X C R 4$ in the hippocampus. To determine whether EE affected the protein expression levels of PKA C- $\alpha$, pCREB, BDNF, SDF-1 and CXCR4, western blotting was conducted on day 34 (Fig. 3A). As presented in Fig. 3B, western blotting identified a significant upregulation of pCREB, BDNF, SDF-1 and CXCR4 expression in the hippocampus following $\mathrm{EE}$; however, there was no significant difference in the protein expression level of PKA C- $\alpha$ between the two groups.

Cognitive function. The Morris water maze was used to assess spatial learning in the rats. EE rats had shorter escape latencies $[\mathrm{F}(1,18)=8.322 ; \mathrm{P}=0.010 ; \mathrm{Fig} .4 \mathrm{~A}]$ and path lengths $[\mathrm{F}(1,18)=6.976 ; \mathrm{P}=0.017$; Fig. 4B] compared with $\mathrm{SE}$ rats; however, there were no significant differences in swimming speeds $[\mathrm{F}(1,18)=0.132 ; \mathrm{P}=0.721 ; \mathrm{Fig}$. $4 \mathrm{C}]$. In the probe trial, there was a significant increase in the number of crossings over the previous position of the platform in the EE group compared

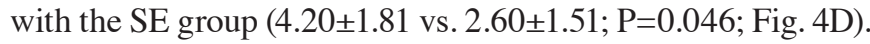
These results suggested that EE rats demonstrated an improved capacity for spatial learning when compared with the SE rats.

\section{Discussion}

The present study demonstrated that 30 days exposure to EE increased the production and differentiation of newly-formed neurons, as well as increasing the dendritic complexity of DCX-positive cells in the DG of adult rats. These structural alterations were accompanied by improved cognitive function in an examination of spatial learning. By contrast, EE did not activate neurogenesis in the SVZ. Furthermore, EE enhanced the protein expression levels of BDNF, pCREB, SDF-1 and CXCR4 in the hippocampus, which potentially contribute to the promotion of hippocampal neurogenesis.

EE provides increased potential for physical activity and complex social stimulation than is achieved under standard conditions (8). In the present study, EE consisted of a large cage with different toys, including tunnels of various shapes for spatial exploration, a running wheel, ladders and swings for motor stimulation, colored balls for visual stimulation, wooden objects of various shapes and texture for sensory perception, and a house for hiding. These toys and their positions were changed once or twice every week to provide a novel environment. In addition, the EE cage encouraged social interaction, as larger numbers of rats (8-12 rats in the present study) were housed together in wider and more spacious cages with exploration chambers $(25,26)$. Currently, no accurate method to quantify environmental impact exists. Xie et al (27) hypothesized that enrichment-induced exercise was the common downstream effect of all enrichment factors, including cage size, the type and quantity of toys, the number of animals and the duration of enrichment. The physical exercise of rats was recorded and measured through the distance moved and velocity achieved, and this was demonstrated to 

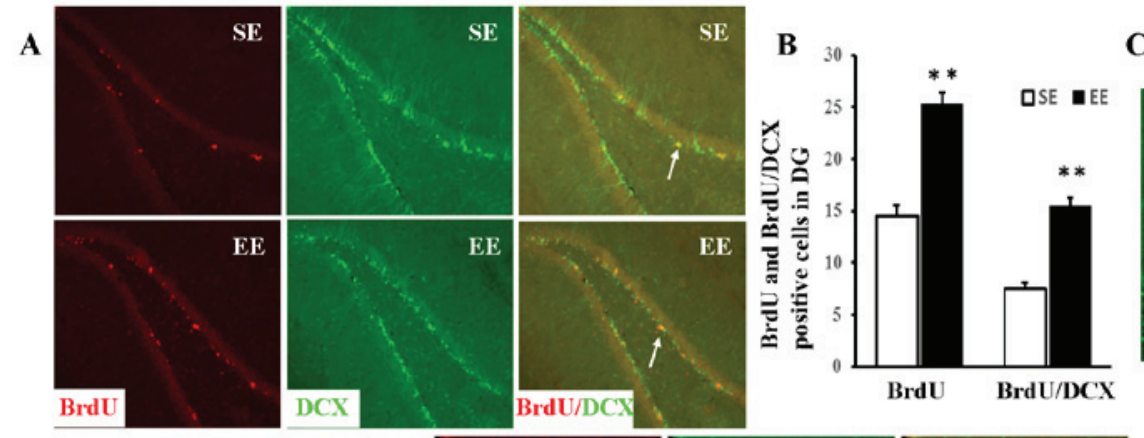

C
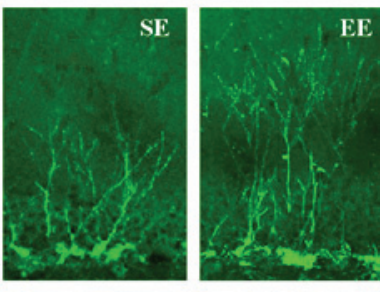

D
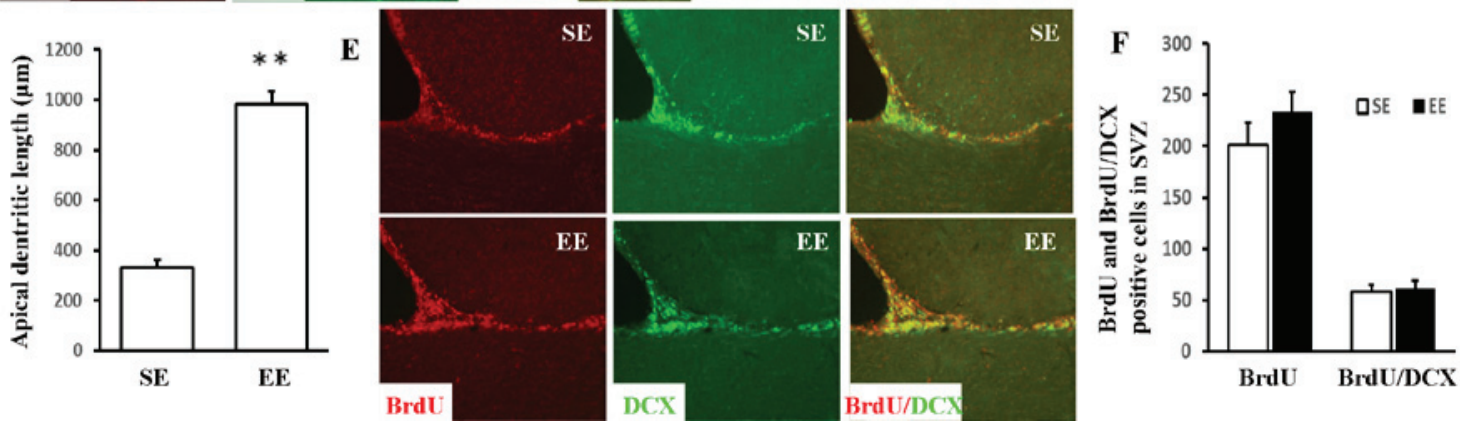

Figure 1. Proliferation and apical dendritic length of newly-formed neurons in the DG and proliferation of newly-formed neurons in the SVZ. (A) Newly-formed neurons in the DG were identified with antibodies recognizing BrdU (red) and DCX (green). White arrows indicate newly-formed neurons. Magnification, x20. (B) Significantly increased numbers of BrdU-positive and BrdU/DCX double-positive cells were observed in the DG of rats in the EE compared with the SE group. (C) The apical dendritic morphology of immature neurons. Magnification, x40. (D) Apical dendritic length was significantly increased in the DG of rats in the EE compared with the SE group. (E) Newly-formed neurons in the SVZ were identified with antibodies recognizing BrdU (red) and DCX (green). Magnification, x20. (F) No significant differences were observed in the numbers of BrdU-positive and BrdU/DCX double-positive cells in the SVZ between the EE and SE groups. ${ }^{* *} \mathrm{P}<0.01$ vs. SE; $\mathrm{n}=6$ per group. BrdU, 5-bromo-2-deoxyuridine; DCX, doublecortin; DG, dentate gyrus; SVZ, subventricular zone; SE, standard environment; EE, environmental enrichment.
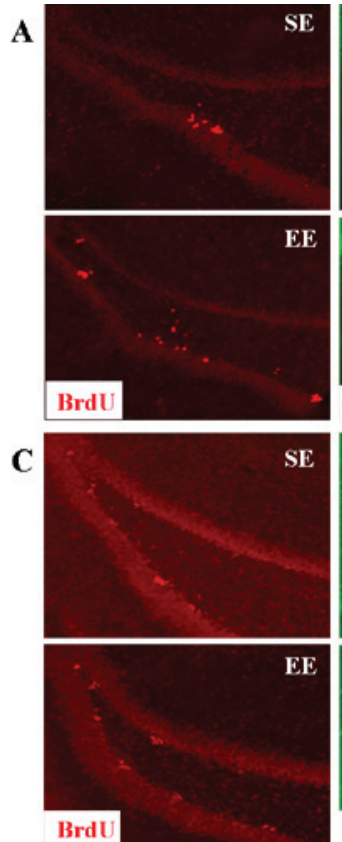
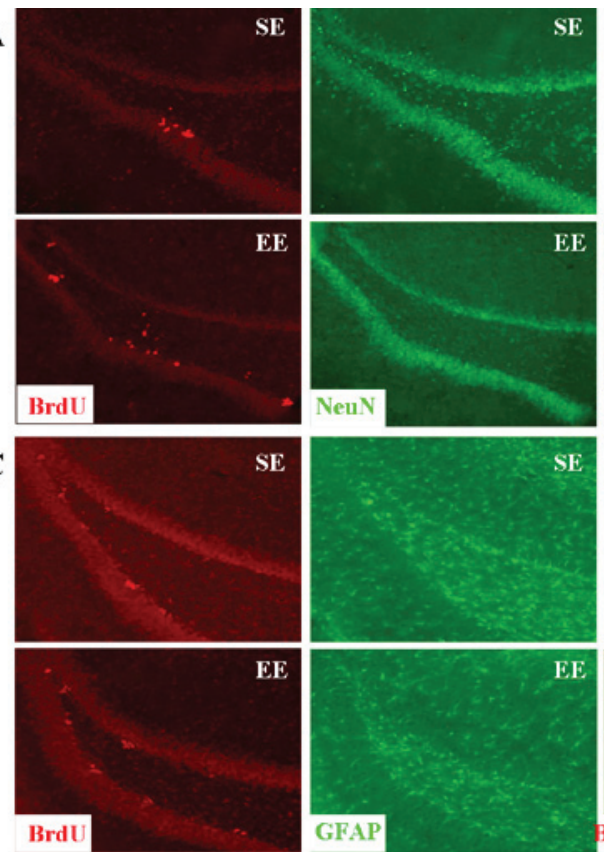
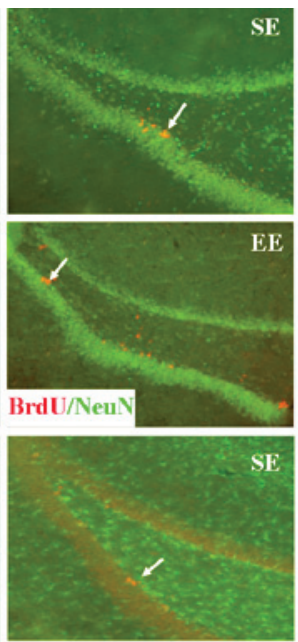

EE

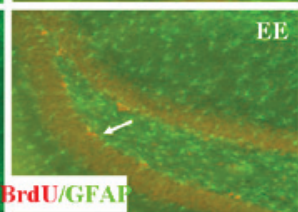

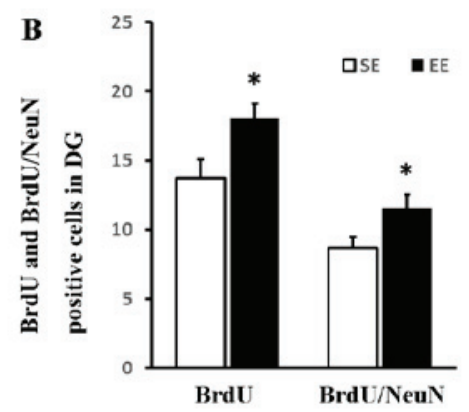

D

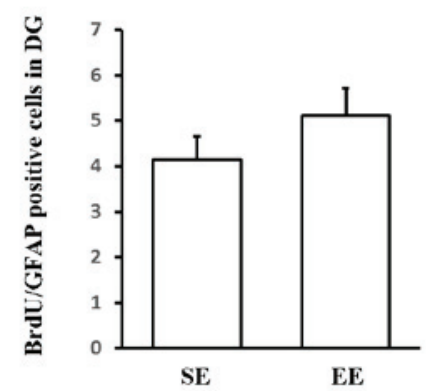

Figure 2. Newly-formed mature neurons and astrocytes in the DG. (A) Newly-formed mature neurons in the DG were identified with antibodies recognizing BrdU (red) and NeuN (green). White arrows indicate newly-formed mature neurons. (B) Increased numbers of BrdU-positive cells and BrdU/NeuN double-positive cells were observed in the EE compared with the SE group. (C) The newly-formed astrocytes in the DG were identified with antibodies recognizing BrdU (red) and GFAP (green). White arrows indicate newly-formed astrocytes. (D) No significant difference in the numbers of BrdU/GFAP double-positive cells were observed between the two groups. Magnification, x20. * $\mathrm{P}<0.05$ vs. SE; n=6 per group. BrdU, 5-bromo-2-deoxyuridine; NeuN, neuronal nuclei; GFAP, glial fibrillary acidic protein; DG, dentate gyrus; SE, standard environment; EE, environmental enrichment.

correlate with the degree of enrichment and the enrichment impact. In addition, Walker and Mason (28) revealed that the degree of enrichment-associated impact was reflected in the physical exercise of animals stimulated by EE. Therefore, 

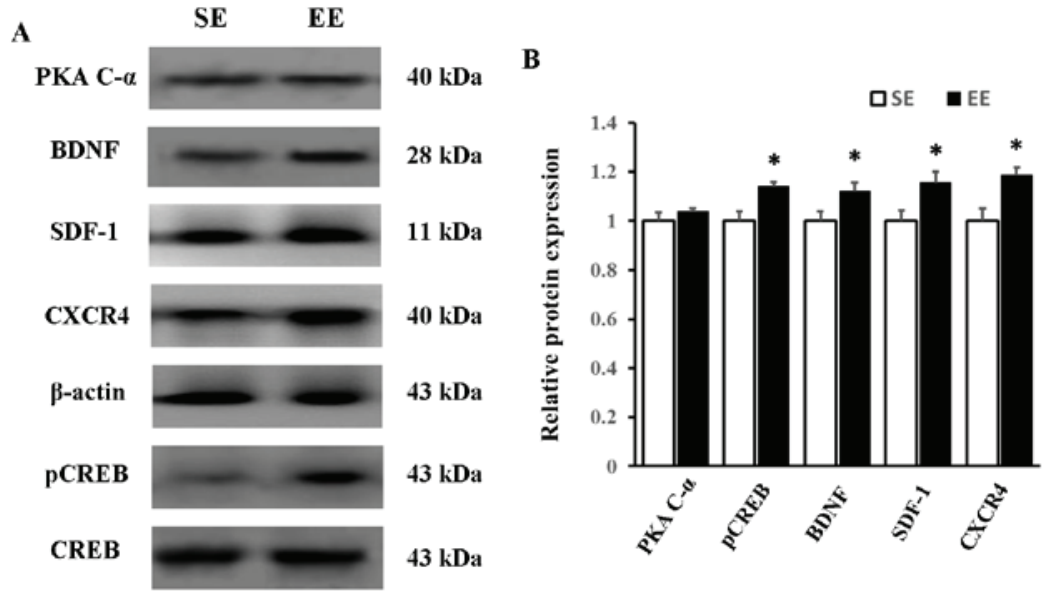

Figure 3. Western blot analysis. (A) Representative immunoblots of PKA C- $\alpha$, BDNF, SDF-1, CXCR4, pCREB and CREB in the hippocampus of rats in the SE and EE groups. (B) Protein expression levels of pCREB, BDNF, SDF-1 and CXCR4 were increased in the EE compared with the SE group, whereas no significant difference was observed in the expression of PKA C- $\alpha$. The protein expression levels of PKA C- $\alpha$, BDNF, SDF- 1 and CXCR4 were normalized to $\beta$-actin expression, and pCREB was normalized to CREB expression. ${ }^{*} \mathrm{P}<0.05$ vs. SE; $\mathrm{n}=6$ per group. PKA $\mathrm{C}-\alpha$, protein kinase A catalytic subunit $\alpha$; BDNF, brain-derived neurotrophic factor; SDF-1, stromal cell-derived factor-1; CXCR4, CXC chemokine receptor 4; CREB, cyclic adenosine monophosphate response element-binding protein; pCREB, phosphorylated CREB; SE, standard environment; EE, environmental enrichment.
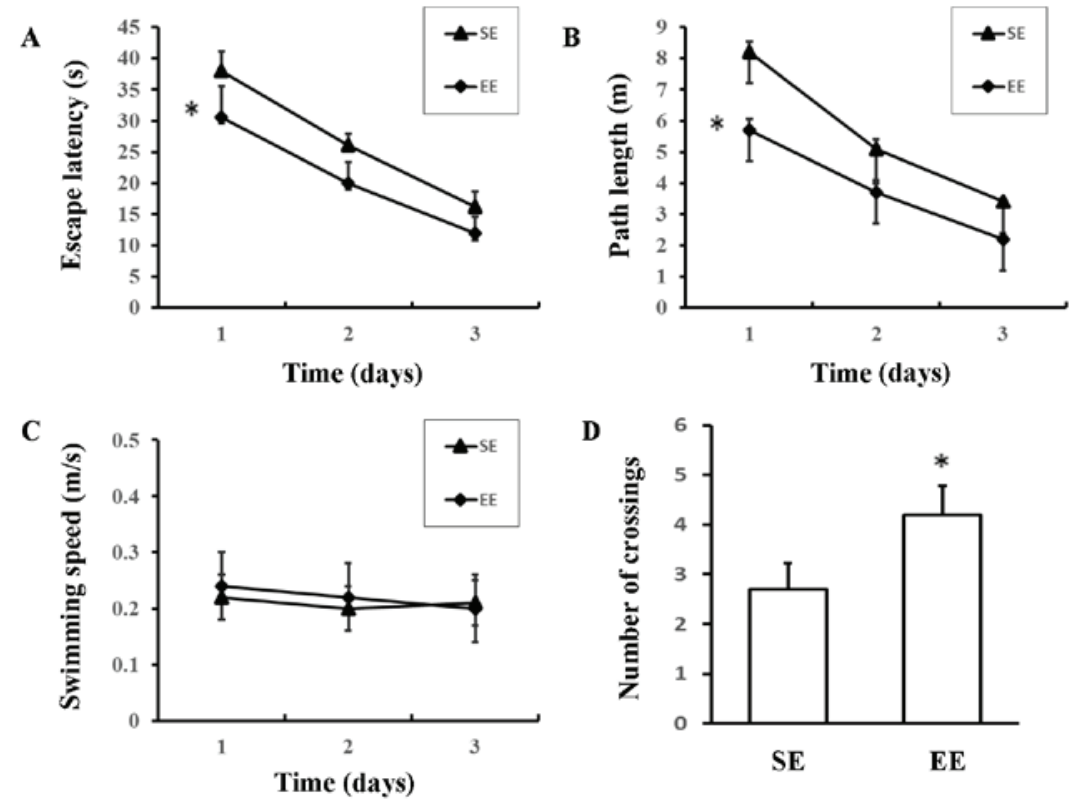

Figure 4. Morris water maze performance of rats in the SE and EE groups. (A) Escape latency and (B) path length were significantly reduced in EE compared with SE rats. (C) No significant differences were observed in swimming speed between the two groups. (D) In the probe trial, performed following the final water maze test, there was a significant increase in the number of crossings over the previous position of the platform in the EE group compared with the SE group. ${ }^{*} \mathrm{P}<0.05$ vs. $\mathrm{SE} ; \mathrm{n}=10$ per group. $\mathrm{SE}$, standard environment; $\mathrm{EE}$, environmental enrichment.

measuring the enrichment-induced exercise of animals may allow quantification of the environmental impact; therefore, this will be performed in any of our future studies. In the present study, the abundant inanimate and social stimulations had a distinct effect on physical exercise, which is known to be beneficial to the central nervous system (29), and the rats with EE performed more enrichment-induced exercise compared with SE rats. The results of the present study are consistent with previous reports that adult rats exposed to EE exhibit improved cognitive functions $(12,30,31)$. Although the underlying mechanisms remain to be elucidated, it is possible that cognitive improvements associated with EE in the normal brain are associated with the enhanced neural plasticity occurring at various levels in the brain. The evidence for enhanced neural plasticity includes neurogenesis, multiple structural changes, increases in neurotrophic factors, and essential proteins and genes involved in neuronal plasticity, as well as alterations in neurotransmitters and receptors $(11,12,32)$.

Previous reports have consistently demonstrated that hippocampal neurogenesis is enhanced by EE. The newly-formed neurons become integrated into the pre-existing hippocampal circuitry and contribute to hippocampal function, at least 
to a certain extent $(12,33)$. However, whether EE alters the differentiation and survival of newly-formed cells remains controversial. Madronal et al (30) observed no differences between EE and SE mice in the numbers of BrdU/NeuN double-positive cells in the DG. By contrast, provision of EE to guinea pigs in the early postnatal period increased cell proliferation and survival, with greater numbers of cells exhibiting a neuronal phenotype compared with controls (34). In the present study, increased numbers of BrdU/NeuN double-positive cells were observed in the DG of the EE, compared with the SE, group; however, no differences were identified in the numbers of BrdU/GFAP double-positive cells between the two groups. Taken together, these results indicate that EE promoted the differentiation of newly-formed cells into neurons rather than astrocytes and enhanced the survival of these novel neurons in the DG.

Previous studies have reported that odor enrichment i.e. an environment in which there are changing sources of odor each day, effectively improved olfactory discrimination learning and enhanced olfactory bulb neurogenesis (35-37). The primary consequences of olfactory stimulation on neurogenesis were the enhanced proliferation and survival of newly-formed cells (35). In support of this, it has been demonstrated, by comparing anosmic and wild-type mice, that a specific sensory input is critical for the survival of newly-formed lateral ventricle wall/olfactory bulb neurons (38). The marked differences between the SVZ and DG regarding the spatial distribution of the components of their neurogenesis systems may explain why neurogenesis in the SVZ was unaffected by $\mathrm{EE}$ in the present study.

There is convincing evidence that PKA/CREB are important in numerous features of neuronal plasticity, including cell proliferation, differentiation, survival and learning $(39,40)$. Certain genes containing cAMP-response element sequences in their promoter regions, particularly BDNF, have been associated with promoting neurogenesis and long-term memory (14). Therefore, the roles of BDNF, pCREB and PKA as mediators of the effects of EE on neurogenesis were evaluated in the present study. It was observed that the activation of BDNF and pCREB protein may link neurogenesis and cognition in EE rats. However, the effect of EE on PKA-linked parameters remains to be elucidated. It has been reported that EE modified the PKA-dependence of hippocampal long-term potentiation and improved hippocampus-dependent memory (41). By contrast, the provision of EE for 30 days in the early postnatal period did not alter immunoreactivity to PKA in the hippocampus (42). In the present study, no significant alterations in PKA C- $\alpha$ protein expression levels were observed, suggesting that the pCREB protein expression level increase following EE may be due to signaling pathways that do not involve PKA. Furthermore, it was demonstrated that provision of EE for 30 days enhanced the protein expression levels of SDF-1 and CXCR4 in the hippocampus; previously, these factors have been implicated in various processes of neurogenesis, including proliferation, differentiation and survival, via stimulation of a cAMP-mediated signaling pathway $(43,44)$. In addition, EE may increase the production of nerve growth factor (45) and induce the expression of the neural cell adhesion molecule (46), which are associated with neurogenesis in the DG. EE has been demonstrated to buffer stress-induced damage in the hippocampus by enhancing the expression of the gene encoding glucocorticoid receptors (47). Thus, it is likely that pCREB, BDNF and SDF-1/CXCR4 are involved in mediating the neuroprotective properties and cognitive effects associated with EE.

In conclusion, the results of the present study demonstrate that EE treatment effectively improved cognitive function and enhanced neurogenesis in the DG of adult rats, which may explain certain effects of EE. The increased protein expression levels of pCREB, BDNF and SDF-1/CXCR4 in the hippocampus may be associated with these beneficial effects. These results may improve understanding of the effects of environment on the brain and provide a theoretical basis for promoting the use of EE in animal facilities.

\section{Acknowledgements}

The present study was supported by the National Natural Science Foundation of China (grant no. 81372104), the Natural Science Foundation of Liaoning Province (grant no. 201202276) and the Program for Liaoning Excellent Talents in University (grant no. LR2013039).

\section{References}

1. Williamson LL, Chao A and Bilbo SD: Environmental enrichment alters glial antigen expression and neuroimmune function in the adult rat hippocampus. Brain Behav Immun 26: 500-510, 2012.

2. Scharfman H, Goodman J, Macleod A, Phani S, Antonelli C and Croll S: Increased neurogenesis and the ectopic granule cells after intrahippocampal BDNF infusion in adult rats. Exp Neurol 192: 348-356, 2005.

3. Serafini G, Hayley S, Pompili M, Dwivedi Y, Brahmachari G, Girardi $\mathrm{P}$ and Amore M: Hippocampal neurogenesis, neurotrophic factors and depression: Possible therapeutic targets? CNS Neurol Disord Drug Targets 13: 1708-1721, 2014.

4. Son Y, Yang M, Kang S, Lee S, Kim J, Kim J, Park S, Kim JS, Jo SK, Jung U, et al: Cranial irradiation regulates CREB-BDNF signaling and variant BDNF transcript levels in the mouse hippocampus. Neurobiol Learn Mem 121: 12-19, 2015.

5. Gage FH, Kempermann G, Palmer TD, Peterson DA and Ray J: Multipotent progenitor cells in the adult dentate gyrus. J Neurobiol 36: 249-266, 1998.

6. van Praag H, Kempermann G and Gage FH: Neural consequences of environmental enrichment. Nat Rev Neurosci 1: 191-198, 2000.

7. Murphy M, Greferath U, Nag N, Nithianantharajah J and Wilson YM: Tracing functional circuits using c-Fos regulated expression of marker genes targeted to neuronal projections. Front Biosci 9: 40-47, 2004

8. Nithianantharajah J and Hannan AJ: Enriched environments, experience-dependent plasticity and disorders of the nervous system. Nat Rev Neurosci 7: 697-709, 2006.

9. Nithianantharajah J, Levis H and Murphy M: Environmental enrichment results in cortical and subcortical changes in levels of synaptophysin and PSD-95 proteins. Neurobiol Learn Mem 81: 200-210, 2004.

10. Pang TY, Stam NC, Nithianantharajah J, Howard ML and Hannan AJ: Differential effects of voluntary physical exercise on behavioral and brain-derived neurotrophic factor expression deficits in Huntington's disease transgenic mice. Neuroscience 141: 569-584, 2006.

11. Leger M, Paizanis E, Dzahini K, Quiedeville A, Bouet V, Cassel JC, Freret T, Schumann-Bard P and Boulouard M: Environmental enrichment duration differentially affects behavior and neuroplasticity in adult mice. Cereb Cortex 25: 4048-4061, 2015.

12. Monteiro BM, Moreira FA, Massensini AR, Moraes MF and Pereira GS: Enriched environment increases neurogenesis and improves social memory persistence in socially isolated adult mice. Hippocampus 24: 239-248, 2014. 
13. Fan D, Li J, Zheng B, Hua L and Zuo Z: Enriched environment attenuates surgery-induced impairment of learning, memory and neurogenesis possibly by preserving BDNF expression. Mol Neurobiol 53: 344-354, 2016.

14. Yang JL, Lin YT, Chuang PC, Bohr VA and Mattson MP: BDNF and exercise enhance neuronal DNA repair by stimulating CREB-mediated production of apurinic/apyrimidinic endonuclease 1. Neuromolecular Med 16: 161-174, 2014.

15. Kuzumaki N, Ikegami D, Tamura R, Hareyama N, Imai S, Narita M, Torigoe K, Niikura K, Takeshima H, Ando T, et al Hippocampal epigenetic modification at the brain-derived neurotrophic factor gene induced by an enriched environment Hippocampus 21: 127-132, 2011.

16. Williams BM, Luo Y, Ward C, Redd K, Gibson R, Kuczaj SA and McCoy JG: Environmental enrichment: Effects on spatial memory and hippocampal CREB immunoreactivity. Physiol Behav 73: 649-658, 2001

17. Zhong L, Yan CH, Lu CQ, Xu J, Huang H and Shen XM: Calmodulin activation is required for the enhancement of hippocampal neurogenesis following environmental enrichment. Neurol Res 31: 707-713, 2009.

18. Marquez-Curtis LA and Janowska-Wieczorek A: Enhancing the migration ability of mesenchymal stromal cells by targeting the SDF-1/CXCR4 axis. Biomed Res Int 2013: 561098, 2013.

19. Cheng $M$ and Qin G: Progenitor cell mobilization and recruitment: SDF-1, CXCR4, $\alpha 4$-integrin and c-kit. Prog Mol Biol Transl Sci 111: 243-264, 2012.

20. Cui L, Qu H, Xiao T, Zhao M, Jolkkonen J and Zhao C: Stromal cell-derived factor- 1 and its receptor CXCR4 in adult neurogenesis after cerebral ischemia. Restor Neurol Neurosci 31: 239-251, 2013

21. Dwinell MB, Ogawa H, Barrett KE and Kagnoff MF: SDF-1/CXCL12 regulates cAMP production and ion transport in intestinal epithelial cells via CXCR4. Am J Physiol Gastrointest Liver Physiol 286: G844-G850, 2004.

22. Odemis V, Moepps B, Gierschik P and Engele J: Interleukin-6 and cAMP induce stromal cell-derived factor- 1 chemotaxis in astroglia by up-regulating CXCR4 cell surface expression. Implications for brain inflammation. J Biol Chem 277: 39801-39808, 2002.

23. Zhang X, Liu T, Zhou Z, Mu X, Song C, Xiao T, Zhao M and Zhao C: Enriched environment altered aberrant hippocampal neurogenesis and improved long-term consequences after temporal lobe epilepsy in adult rats. J Mol Neurosci 56: 409-421, 2015.

24. Qu HL, Zhao M, Zhao SS, Xiao T, Song CG, Cao YP, Jolkkonen $\mathrm{J}$ and Zhao CS: Forced limb-use enhanced neurogenesis and behavioral recovery after stroke in the aged rats Neuroscience 286: 316-324, 2015.

25. Dhanushkodi A and Shetty AK: Is exposure to enriched environment beneficial for functional post-lesional recovery in temporal lobe epilepsy? Neurosci Biobehav Rev 32: 657-674, 2008

26. Fares RP, Belmeguenai A, Sanchez PE, Kouchi HY, Bodennec J, Morales A, Georges B, Bonnet C, Bouvard S, Sloviter RS and Bezin L: Standardized environmental enrichment supports enhanced brain plasticity in healthy rats and prevents cognitive impairment in epileptic rats. PloS One 8: e53888, 2013.

27. Xie H, Wu Y, Jia J, Liu G, Zhang Q, Yu K, Guo Z, Shen L and $\mathrm{Hu}$ R: Enrichment-induced exercise to quantify the effect of different housing conditions: A tool to standardize enriched environment protocols. Behav Brain Res 249: 81-89, 2013.

28. Walker MD and Mason G: Female C57BL/6 mice show consistent individual differences in spontaneous interaction with environmental enrichment that are predicted by neophobia. Behav Brain Res 224: 207-212, 2011

29. Ambrogini P, Lattanzi D, Ciuffoli S, Betti M, Fanelli M and Cuppini R: Physical exercise and environment exploration affect synaptogenesis in adult-generated neurons in the rat dentate gyrus: Possible role of BDNF. Brain Res 1534: 1-12, 2013.
30. Madroñal N, López-Aracil C, Rangel A, del Río JA Delgado-García JM and Gruart A: Effects of enriched physical and social environments on motor performance, associative learning and hippocampal neurogenesis in mice. PloS One 5: e11130, 2010.

31. Silva CF, Duarte FS, Lima TC and de Oliveira CL: Effects of social isolation and enriched environment on behavior of adult Swiss mice do not require hippocampal neurogenesis. Behav Brain Res 225: 85-90, 2011.

32. Alvarez PS, Simão F, Hemb M, Xavier LL and Nunes ML: Effects of undernourishment, recurrent seizures and enriched environment during early life in hippocampal morphology. Int J Dev Neurosci 33: 81-87, 2014.

33. Shors TJ, Townsend DA, Zhao M, Kozorovitskiy Y and Gould E: Neurogenesis may relate to some but not all types of hippocampal-dependent learning. Hippocampus 12: 578-584, 2002.

34. Rizzi S, Bianchi P, Guidi S, Ciani E and Bartesaghi R: Impact of environmental enrichment on neurogenesis in the dentate gyrus during the early postnatal period. Brain Res 1415: 23-33, 2011.

35. Rochefort C, Gheusi G, Vincent JD and Lledo PM: Enriched odor exposure increases the number of newborn neurons in the adult olfactory bulb and improves odor memory. J Neurosci 22 : 2679-2689, 2002

36. Bonzano S, Bovetti S, Fasolo A, Peretto P and De Marchis S: Odour enrichment increases adult-born dopaminergic neurons in the mouse olfactory bulb. Eur J Neurosci 40: 3450-3457, 2014.

37. Martončiková M, Lievajová K, Orendáčová J, Blaško J and Račeková E: Odor enrichment influences neurogenesis in the rostral migratory stream of young rats. Acta Histochem 113: 326-332, 2011.

38. Petreanu L and Alvarez-Buylla A: Maturation and death of adult-born olfactory bulb granule neurons: Role of olfaction. J Neurosci 22: 6106-6113, 2002.

39. Carlezon WA Jr, Duman RS and Nestler EJ: The many faces of CREB. Trends Neurosci 28: 436-445, 2005.

40. Li QQ, Shi GX, Yang JW, Li ZX, Zhang ZH, He T, Wang J, Liu LY and Liu CZ: Hippocampal cAMP/PKA/CREB is required for neuroprotective effect of acupuncture. Physiol Behav 139: 482-490, 2015.

41. Duffy SN, Craddock KJ, Abel T and Nguyen PV: Environmental enrichment modifies the PKA-dependence of hippocampal LTP and improves hippocampus-dependent memory. Learn Mem 8: 26-34, 2001.

42. Xie T, Wang WP, Jia LJ, Mao ZF, Qu ZZ, Luan SQ and Kan MC: Environmental enrichment restores cognitive deficits induced by prenatal maternal seizure. Brain Res 1470: 80-88, 2012.

43. Chalasani SH, Baribaud F, Coughlan CM, Sunshine MJ, Lee VM, Doms RW, Littman DR and Raper JA: The chemokine stromal cell-derived factor- 1 promotes the survival of embryonic retinal ganglion cells. J Neurosci 23: 4601-4612, 2003.

44. Opatz J, Küry P, Schiwy N, Järve A, Estrada V, Brazda N, Bosse F and Müller HW: SDF-1 stimulates neurite growth on inhibitory CNS myelin. Mol Cell Neurosci 40: 293-300, 2009.

45. Birch AM, McGarry NB and Kelly AM: Short-term environmental enrichment, in the absence of exercise, improves memory, and increases NGF concentration, early neuronal survival, and synaptogenesis in the dentate gyrus in a time-dependent manner. Hippocampus 23: 437-450, 2013.

46. Young D, Lawlor PA, Leone P, Dragunow M and During MJ: Environmental enrichment inhibits spontaneous apoptosis, prevents seizures and is neuroprotective. Nat Med 5: 448-453, 1999.

47. Zhang L, Zhang J, Sun H, Liu H, Yang Y and Yao Z: Exposure to enriched environment restores the mRNA expression of mineralocorticoid and glucocorticoid receptors in the hippocampus and ameliorates depressive-like symptoms in chronically stressed rats. Curr Neurovasc Res 8: 286-293, 2011. 